\title{
Biportal Endoscopic Lumbar Interbody Fusion: Review of Current Evidence and the Literature
}

\author{
Man-Kyu Park, Sang-Kyu Son \\ Department of Neurosurgery, ParkWeonWook Hospital, Busan, Republic of Korea
}

Corresponding Author: Sang-Kyu Son, MD Department of Neurosurgery, ParkWeonWook Hospital, 4, Gwangan-ro, Suyeong-gu, Busan, 48298, Republic of Korea

Tel: $+82-70-4000-765$

Fax: +82-51-751-5287

E-mail: jihak3@gmail.com

Received: February 02, 2021

Revised: March 20, 2021

Accepted: April 04, 2021

\begin{abstract}
Several studies report that biportal endoscopic lumbar interbody fusion (ELIF) demonstrate various advantages such as favorable clinical outcomes, less postoperative back pain, and shorter hospital stays compared to open PLIF/MIS TLIF. Even though biportal ELIF has demonstrated several advantages, limitations are also associated with the technique, including longer operation time, limited indications, and a lack of multicenter randomized controlled trials that directly compare other fusion techniques with biportal ELIF. The objective of this review was to evaluate previously published articles and summarize current evidence on biportal ELIF regarding its indications, surgical technique, complications and comparative results with open PLIF/MIS TLIF.
\end{abstract}

Key Words: Biportal, Endoscopy, Interbody fusion, Minimal invasive surgery, Lumbar

\section{INTRODUCTION}

While conventional posterior lumbar interbody fusion (PLIF) or transforaminal lumbar interbody fusion (TLIF) techniques have shown good clinical and radiological outcomes for lumbar degenerative disease, they have also been associated with paraspinal muscle injury from subperiosteal dissections and retraction, which can lead to increased postoperative back pain and muscle atrophy ${ }^{2,9,18,19)}$. Threfore, recent techniques for endoscopic lumbar interbody fusion (ELIF) have been developed, which are associated with minimal muscle injury to posterior musculo-ligamentous structures, good clinical outcomes, and satisfactory fusion rates, ${ }^{1,-6,8,8,10-12,16,17,21-23)}$. There are several studies on uniportal ELIF, 12,17,21-23), and biportal ELIF has been published by several investigators ${ }^{46,10,11,16)}$. The lumbar interbody fusion under unilateral biportal endoscpy (UBE) guidance, uses a separate endoscopic portal and working portal. Unlike uniportal $E L I F$, independent movement of the endoscope and surgical instruments is possible, and the working portal is used only for surgical instruments not though the working cannula. As a result, biportal ELIF can achieve direct ipsilateral and contralateral decompression similar to MIS TLF with fewer limitations of motion and vision ${ }^{4-6,10,11,16)}$. Moreover, biportal ELIF has advantages in terms of minimal invasiveness ${ }^{5,11,16)}$.

Since its introduction, several studies report that biportal ELIF show various kinds of advantages such as favorable clinical outcomes, less postoperative back pain, and a shorter hospital stays compared to open PLIF/MIS TLF ${ }^{5,6,11,16)}$. The objective of this review was to evaluate previously published articles and summarize the current evidence on biportal ELIF regarding its indications, surgical technique, complications and comparative results with open PLIF/MIS TLIF.

\section{METHODS}

\section{Selections of Studies}

A literature search was performed using the National Center for Biotechnology Information databases using PubMed/MEDLINE search engines. Keywords included the following: "Biportal endoscopic lumbar interbody fusion," "Unilateral biportal endoscopy," "Lumbar," and "Interbody fusion." Medline and Scopus databases were used for identifying relevant studies published in English. While reviewing the literature, articles on biportal ELIF were retrieved using the above-described search. There were three case-control studies, two case series articles, and one technical note and review article. The characteristics of these studies are summarized in Table 1.

\section{RESULTS}

\section{Surgical Indications and Contraindication for Biportal Endoscopic Lumbar Interbody Fusion}

Broadly, the indications of biportal ELIF are similar to those 
for MIS PLIF/TLIF ${ }^{5,6,16)}$. According to previous articles, the common indications for biportal ELIF are as follows: (1) grade 1 or 2 degenerative or isthmic spondylolisthesis, (2) recurrent disc herniation, or (3) central or foraminal stenosis with instability.

Similar contraindications exist for MIS PLIF/TLIF as contraindications for biportal ELIF, including the following: infection, spondylodiscitis, vertebral fractures, congenital spinal deformity, or high-grade spondylolisthesis (grade 3 or 4$)^{5,6,16}$ ).

\section{Operative Technique}

There are minor variations in the biportal ELIF technique amongst practitioners, and the surgical technique has been previously described ${ }^{4-6,10,11,16)}$. Briefly, patients are prepared in a prone position under general or epidural anesthesia. A skin incision for the endoscopic portal is made cranially; another skin incision for the working portal is made caudally. The target point is identified using an anteroposterior (AP) view of C-arm fluoroscopy as the lower part of the cranial lamina. Two incisions are made about $3 \mathrm{~cm}$ apart, with the center being the lower part of the cranial lamina (Figure 1). Each incision used for percutaneous pedicle screw insertion is placed at the central portion of the cranial and caudal pedicles. The endoscope and retractor are positioned in each portal, and the initial submuscular working space is made under endoscopic guidance above the cranial lamina. Unilateral laminectomy is completed and contralateral decompression is made through a sublaminar approach wide enough for bilateral decompression (Figure 2). Multiple osteotomies are done to remove the ipsilateral inferior articular process and the superior articular process for cage insertion (Figure 3A). Contralateral facetectomy through a sublaminar approach is better to achieve reduced spondylolisthesis or to restore disc height (Figure 3B). Meticulous endplate preparation is performed under endoscopic guidance after exposing the ipsilateral disc space (Figure 4). Using a freer elevator, the cartilaginous endplate can be easily detached from the osseous endplate. Endplate preparation using endoscopic guidance can reduce the likelihood of endplate injury. Autologous and allogenous bone chips are inserted into the disc space after completion of endplate preparation (Figure 5A). A cage is then inserted with a specialized retractor under fluoroscopic guidance to protect the thecal sac and nerve root (Figure 5B). After cage insertion, the procedure is completed with percutaneously pedicle screws under fluoroscopic guidance (Figure 6).

Table 1. Summary of included studies on biportal endoscopic lumbar interbody fusion

\begin{tabular}{|c|c|c|c|c|c|c|c|}
\hline & Study & Study design & No.of patients & Average Age (Yr) & Follow-up (mo) & Operative time (min) & Operative level \\
\hline 1 & Heo et al, 2017 & Case series & 69 & $71.2 \pm 7.8$ & $13.5 \pm 7.1$ & $165.8 \pm 25.5$ & Single \\
\hline 2 & Kim and Choi, 2018 & Case series & 14 & $68.7 \pm 8.5$ & 2 & $169 \pm 10$ & Single \\
\hline 3 & Heo and Park, 20195) & Case control & $\begin{array}{l}\text { Biportal ELIF: } 23 \\
\text { MIS TLIF: } 46\end{array}$ & $61.4 \pm 9.4$ & 12 & $\begin{array}{l}\text { Biportal: } 152.4 \pm 9.6 \\
\text { MIS TLIF: } 122.4 \pm 13.1\end{array}$ & Single \\
\hline 4 & Park et al, 2019 & Case control & $\begin{array}{l}\text { Biportal ELIF: } 71 \\
\text { Open PLIF: } 70\end{array}$ & $68 \pm 8$ & $17.1 \pm 4.9$ & $\begin{array}{l}\text { Biportal: } 158.2 \pm 26.7 \\
\text { Open PLIF: } 136.6 \pm 21.5\end{array}$ & Single \\
\hline 5 & Kim et al, 2020 & Case control & $\begin{array}{l}\text { Biportal ELIF: } 32 \\
\text { MIS TLIF: } 55\end{array}$ & $70.5 \pm 8.3$ & 18.4 & $\begin{array}{l}\text { Biportal: } 169.5 \pm 24.9 \\
\text { MIS TLIF: } 173 \pm 47.1\end{array}$ & Single \\
\hline 6 & Heo et al, 2020 & $\begin{array}{l}\text { Technical note } \\
\text { and review }\end{array}$ & NA & NA & NA & NA & Single \\
\hline
\end{tabular}

ELIF: Endoscopic lumbar interbody fusion, TLIF: transforaminal lumbar interbody fusion, NA: not applicable
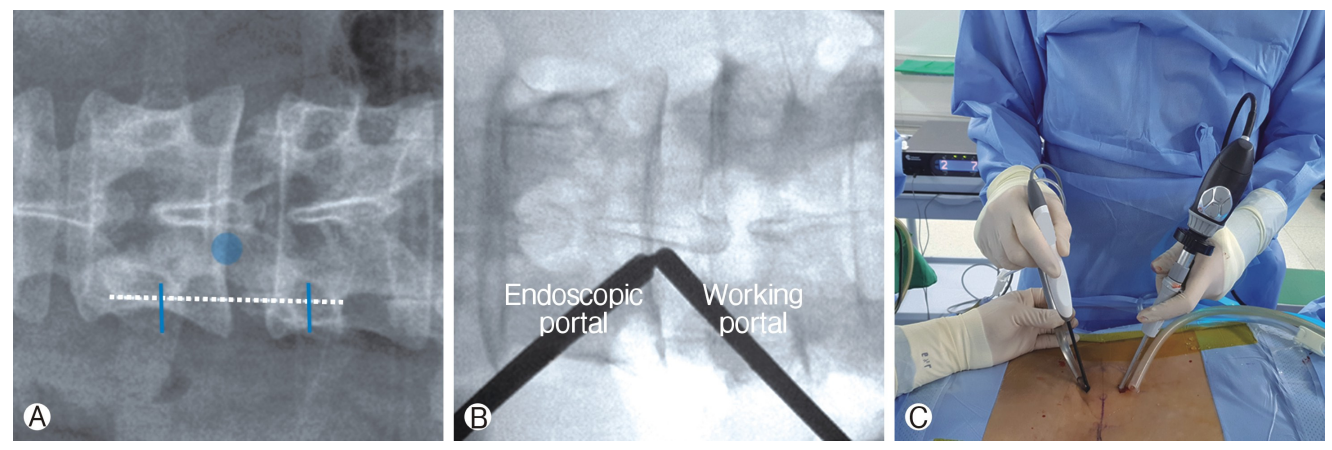

Figure 1. (A): Skin incision and target point under the fluoroscopic AP view. The target point (blue circle) is the lower part of the cranial lamina. The 2 incisions (blue line) are about $3 \mathrm{~cm}$ apart, with the center being the lower part of the cranial lamina. Each incision is placed at the central portion of the cranial and caudal pedicles (white dotted line). (B): A scope through the left incision and a retractor and a instrument through the right incision. (C): Biportal endoscopic lumbar interbody fusion done through space created by a semi-tubular retractor. 

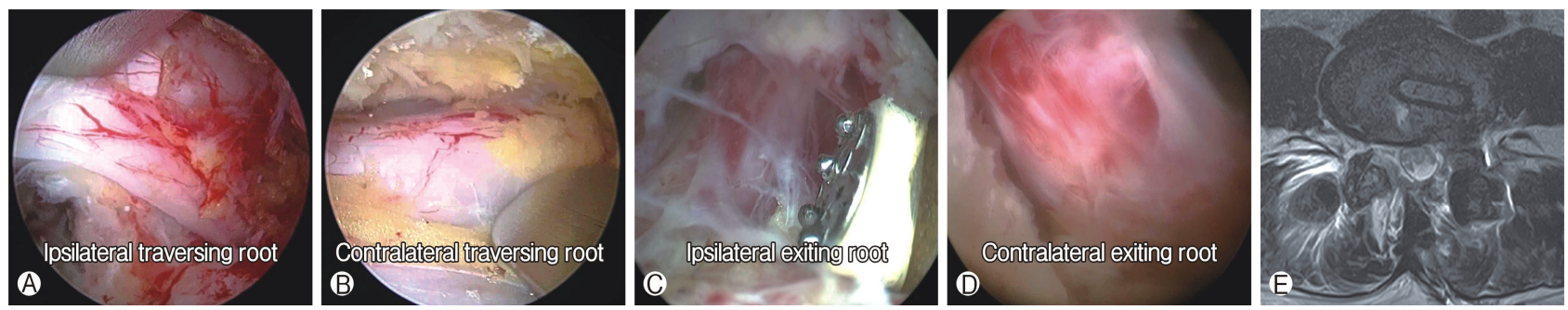

Figure 2. Endoscopic view of complete decompression after biportal endoscopic lumbar interbody fusion. The ipsilateral traversing the nerve root $(A)$, contralateral traversing nerve root $(B)$, ipsilateral exiting nerve root $(C)$ and contralateral exiting nerve root (D). Postoperative magnetic resonance image, axial plane (E).
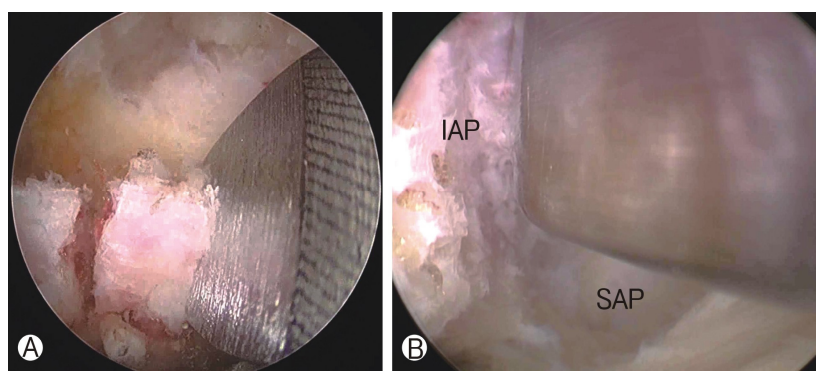

Figure 3. Endoscopic view of facetectomy. (A): Removal of ipsilateral inferior articular process using an osteotome. (B): Removal of contralateral interior articular process using an osteotome.
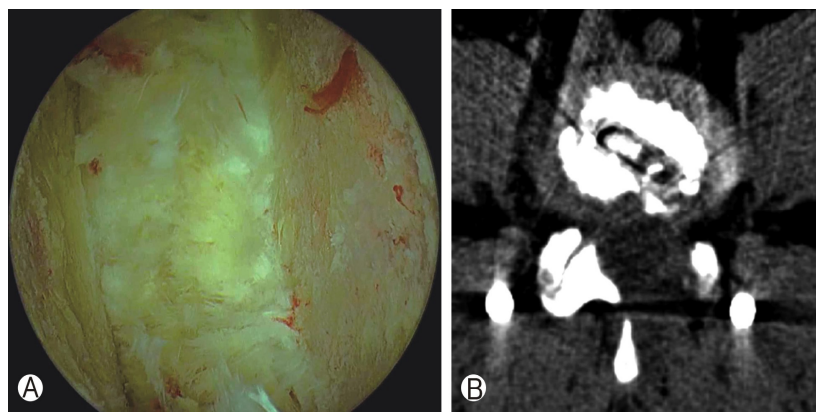

Figure 4. (A): Endoscopic view of meticulous endplate preparation. (B): Postoperative axial CT show the dimension of the endplate preparation after biportal endoscopic lumbar interbody fusion.
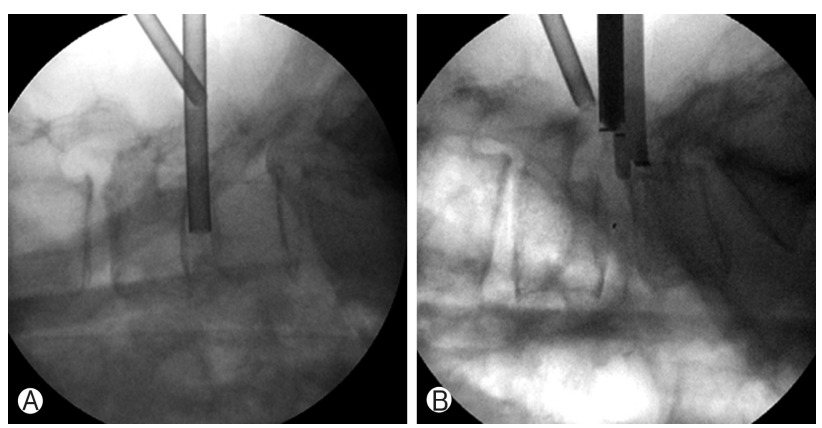

Figure 5. (A): Autologous and allogenous bone chips are inserted into the disc space using a funnel. (B): A cage is then inserted with a specialized retractor under fluoroscopic guidance to protect the thecal sac and nerve root.
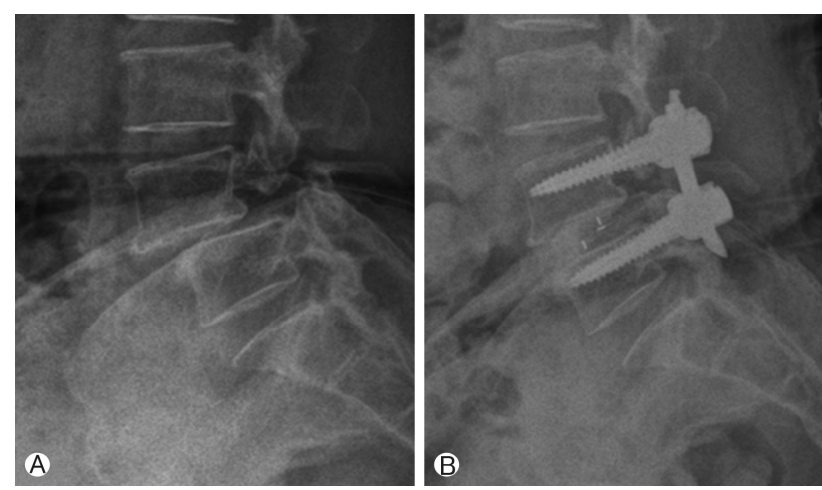

Figure 6. Preoperative simple lateral radiography image of isthmic spondylolisthesis at L4-5 (A). The postoperative simple lateral radiography image shows a reduction in spondylolisthesis (B).

\section{Clinical Outcome}

Operative data and clinical outcomes are summarized in Table 2. The outcome measurements were the visual analog scale (VAS) for back and leg pain, the Oswestry Disability Index (ODI) score and Macnab criteria. The follow-up period was over 12 months after surgery in 4 of the 5 articles ${ }^{5,6,11,16)}$. The follow-up period for the other article was 2 months after surgery ${ }^{10)}$. Outcome measures, as scored by VAS for back and leg pain and ODI improved significantly in all articles after biportal ELIF. In these studies, mean preoperative VAS scores varied from 6.6 to 8.1 for leg pain and from 6.0 to 6.2 for back pain, and final follow-up mean VAS scores varied from 1.6 to 3.6 for leg pain and from 1.8 to 3.1 for back pain. In all these studies, ODI scores significantly improved after biportal ELF. The mean operative ODI score ranged from 45.7 to 68.1 , whereas the postoperative mean ODI score at the final follow-up ranged from 15.4 to 32.7. Clinical outcomes showed that biportal ELIF achieved clinically significant improvement with a reduction greater than 2 points in VAS score and an increase of greater than 20\% in ODI score ${ }^{14)}$. More importantly, the three comparative studies showed that postoperative back pain immediately following surgery was significantly lower than that for open PLIF/MIS TLIF, 11,16).

\section{Fusion Rates}

The final goal of lumbar interbody fusion surgeries is solid 
Man-Kyu Park, et al.

Table 2. Summary of clinical outcome, fusion rate, complications and blood loss of biportal endoscopic lumbar interbody fusion

\begin{tabular}{|c|c|c|c|c|c|}
\hline Study & $\begin{array}{l}\text { Outcome variable } \\
\text {-Preoperatively } \\
\end{array}$ & $\begin{array}{l}\text { Outcome variable } \\
\text {-Postoperatively }\end{array}$ & Fusion rate & Complication & Blood loss \\
\hline $\begin{array}{l}\text { Heo et al, } \\
2017^{6)}\end{array}$ & $\begin{array}{l}\text { ODI: } 45.65 \\
\text { VAS(L): } 8.12\end{array}$ & $\begin{array}{l}\text { ODI: } 15.41 \\
\text { VAS(L): } 2.79\end{array}$ & NA & $\begin{array}{l}5(7.2 \%) \\
\text { Dural tear: } 2 \\
\text { Postop hematoma: } 3\end{array}$ & $85.5 \pm 19.4(\mathrm{ml})$ \\
\hline $\begin{array}{l}\text { Kim and Choi, } \\
2018^{10)}\end{array}$ & VAS:7.4 & VAS:2.7 (2M) & NA & $\begin{array}{l}2(14.3 \%) \\
\text { L5 paralysis: } 1 \\
\text { Dura tear: } 1\end{array}$ & $74 \pm 9(\mathrm{ml})$ \\
\hline $\begin{array}{l}\text { Heo and Park, } \\
2019^{5)}\end{array}$ & $\begin{array}{l}\text { ODI: } 57.8 \\
\text { VAS(L): } 8.1\end{array}$ & $\begin{array}{l}\text { ODI: } 21.8 \\
\operatorname{VAS}(B): 4.2 / 2.8 / 2.4 \\
\operatorname{VAS}(L): 2.5\end{array}$ & $\begin{array}{l}\text { Biportal: } 78.3 \% \\
\text { MIS TLIF: } 73.9 \%\end{array}$ & $\begin{array}{l}\text { Biportal: } 1 \text { (4.3\%) } \\
\text { Postop hematoma:1 } \\
\text { MIS TLIF: } 6 \text { (13.0\%) } \\
\text { Postop hematoma:1 } \\
\text { Dural tear:1 } \\
\text { Infection: } 1 \\
\text { DVT: } 1 \\
\text { Cage subsidence: } 2\end{array}$ & $\begin{array}{l}\text { Biportal } 190.3 \pm 31.0 \\
\quad(\mathrm{ml}) \\
\text { MIS TLIF } 289.3 \pm 58.5 \\
\quad(\mathrm{ml})\end{array}$ \\
\hline $\begin{array}{l}\text { Park et al, } \\
20191^{5)}\end{array}$ & $\begin{array}{l}\text { ODI: } 61.9 \\
\operatorname{VAS}(B): 6.0 \\
\operatorname{VAS}(L): 6.6\end{array}$ & $\begin{array}{l}\text { ODI: } 32.7 \\
\operatorname{VAS}(B): 3.8(1 \mathrm{~W}) / 3.1(12 \mathrm{M}) \\
\operatorname{VAS}(\mathrm{L}): 3.6(1 \mathrm{~W}) / 3.6(12 \mathrm{M})\end{array}$ & $\begin{array}{l}\text { Biportal: } 95.1 \% \\
\text { Open PLIF: } 90.0 \%\end{array}$ & $\begin{array}{l}\text { Biportal: } 5 \text { (7.0\%) } \\
\text { Dural tear: } 3 \\
\text { Postop hematoma: } 1 \\
\text { Infection: } 1 \\
\text { Open TLIF: } 6(8.6 \%) \\
\text { Dural tear: } 2 \\
\text { Nerve root injury: } 1 \\
\text { Postop heamtoma: } 1 \\
\text { Infection: } 2\end{array}$ & $\begin{array}{l}\text { Biportal: transfusion } \\
\text { 0\% } \\
\text { Open PLIF: } \\
\text { Transfusion } 18.6 \%\end{array}$ \\
\hline $\begin{array}{l}\text { Kim et al, } \\
2020^{11)}\end{array}$ & $\begin{array}{l}\text { ODI: } 68.1 \\
\operatorname{VAS}(B): 6.2 \\
\operatorname{VAS}(L): 7.9\end{array}$ & $\begin{array}{l}\text { ODI: } 31.5(2 \mathrm{~W}) / 26.6(2 \mathrm{M}) / 15.6(12 \mathrm{M}) \\
\operatorname{VAS}(\mathrm{B}): 3.1(2 \mathrm{~W}) / 2.4(2 \mathrm{M}) / 1.8(12 \mathrm{M}) \\
\operatorname{VAS}(\mathrm{L}): 3.3(2 \mathrm{~W}) / 2.4(2 \mathrm{M}) / 1.6(12 \mathrm{M})\end{array}$ & $\begin{array}{l}\text { Biportal: } 93.7 \% \\
\text { MIS TLIF: } 92.7 \%\end{array}$ & $\begin{array}{l}\text { Biportal: } 2 \text { (6.3\%) } \\
\text { Postop hematoma: } 1 \\
\text { Transient palsy: } 1 \\
\text { MIS TLIF: } 3 \text { (5.5\%) } \\
\text { Postop hematoma: } 1 \\
\text { Transient palsy: } 2\end{array}$ & NA \\
\hline
\end{tabular}

ODI: Oswestry Disability Index, VAS: Visual analog scale, L: leg, B: back, M: month, W: week, TLIF: Transforaminal lumbar interbody fusion, PLIF: Posterior lumbar interbody fusion, NA: not applicable

fusion. Among the studies examined, 3 were comparative studies with open PLIF/MIS TLIF on fusion rate ${ }^{5,11,16)}$. Radiography at the 1-year follow-up was used to estimate the fusion rate in these articles. All articles on biportal ELIF have shown good fusion rates. However, there was a large variance in the fusion rate from $78.3 \%$ to $95.1 \%$ across the studies. Comparative studies between biportal ELIF and open PLIF or MIS TLIF found similar fusion rates.

\section{Complications}

Complications for biportal ELIF have included postoperative hematoma, dural tear, infection and nerve root injury in previously published articles $5,6,10,11,16)$. Three articles compared the complications rates with conventional PLIF or MIS TLIF, and there was no significant difference. The overall rate of complications ranged from $4.3 \%$ to $14.3 \%$ for biportal ELIF, and from $5.5 \%$ to $13.0 \%$ for open PLIF/ MIS TLIF.

\section{Operative Time}

All the studies examined used single-level biportal ELIF. The mean operation times for biportal ELIF versus open PLIF/MIS TUF ranged from 152.4 to 169.5 minutes and 122.4 to 173 minutes, respectively. The mean operation times for biportal ELIF were longer than that for open PLIF/MIS TLIF.

\section{Blood loss}

Two comparative articles compared the blood loss for biportal ELIF with that for open PLIF/MIS TLIF. They found a significant difference in intraoperative blood loss (biportal ELIF: $190.3 \mathrm{~mL}$, MIS TLIF: $289 \mathrm{~mL})^{5}$ and statistically significant reductions in transfusion were noted with biportal ELIF compared with open PLIF ${ }^{16)}$. 


\section{DISCUSSION}

\section{Biportal Endoscopic Lumbar Interbody Fusion}

Conventional or MIS PLIF/TLIF have been demonstrated to be good surgical techniques for treating degenerative lumbar spinal disease ${ }^{13,20)}$. However, there are disadvantages associated with these techniques, such as back muscle atrophy and postoperative back pain arising from muscle dissection and retraction $^{29,18,19)}$. On the other hand, similar fusion rates and clinical outcomes have been reported with biportal ELIF, while showing reduced blood loss and less postoperative back pain, which may allow faster recovery after the operation ${ }^{5,11,16)}$.

Six articles on biportal ELIF have been published (Table 1, 2). Two articles described the technique and reported preliminary clinical results ${ }^{6,10)}$. One article was a technical note and review article $^{4)}$. This article focused on the technical aspect of biportal ELIF. The other 3 articles presented comparative studies between biportal ELIF and open PLIF or MIS TLIF ${ }^{5,11,16)}$.

Heo et al. ${ }^{6)}$ followed with a description of biportal ELIF for single-level degenerative disease in 69 patients. They demonstrated a statistically significant improvement in ODI $(p<0.05)$ and VAS $(p<0.05)$ with biportal ELIF with an average follow-up of 13.5 months. The complications included 5 cases of perioperative complications including ( 2 patients with dural tear and, 3 postoperative hematoma. None of the complications required revisions. Similarly, Kim and $\mathrm{Choi}^{10)}$ reported improved VAS scores at postoperative 2 months in 14 patients who underwent a single-level biportal ELIF. The authors found that 2 of 14 patients had complications including 1 case of dural tear and 1 case of L5 root palsy.

Three articles were comparative studies between conventional PLIF or MIS TLIF and biportal ELIF. Park et al. ${ }^{16)}$ retrospectively reviewed 71 patients who underwent biportal ELIF and open PLIF (70 patients) for single-level disease. The authors demonstrated that biportal ELIF had statistically significant improvements in back and leg VAS and ODI, but were not significantly different between the 2 groups at 1-year postoperatively (mean follow-up: 17 months). More importantly, postoperative back pain at 1 week was significantly higher with open PLIF than with biportal ELIF. They found a significant difference in mean operation time (biportal: $158 \mathrm{~min}$, open: $137 \mathrm{~min}$ ), and a statistically significant reduction in transfusion was noted with biportal ELIF $(p<0.001)$. The incidences of surgical complications were not significantly different between the 2 groups. No statistically significant difference was seen in the fusion rates between biportal ELIF (95.1\%) and open PLIF (90.0\%).

A study by $\mathrm{HeO}$ and Park ${ }^{5}$ demonstrated the advantages biportal ELIF when compared with MIS TLIF. This study compared 23 patients who underwent 1- level biportal ELIF with a cohort of 46 patients who underwent a 1- level MIS TLIF. At the 1-year follow-up, the authors demonstrated that biportal ELIF had good clinical outcomes for VAS and ODI. The biportal ELIF group had less intraoperative blood loss $(p<0.05)$ and postoperative back pain on day 1 and day $2(\mathrm{p}<0.05)$ compared with MIS TLF. Radiographic fusion was shown in 18 of 23 patients (78.3\%) at 12 months, which was not significantly different in comparison with MIS TLIF (73.9\%). Further comparative studies of biportal ELIF with MIS TLIF were performed by Kim et $\mathrm{al}^{111}$. Recently, Kim et al. demonstrated the superiority of biportal ELIF to MIS TLIF for 1-level fusion in a retrospective cohort study of 87 patients (32 biportal ELIF, 55 MIS TLIF). At a mean follow-up of 18.4 years, they reported a statistically significant improvement in ODI and VAS for back and leg for biportal ELIF. As anticipated, biportal ELIF demonstrated reduced back pain in the second week following surgery compared to MIS TLIF ( $p=0.001)$. The difference in the fusion rates between biportal ELIF (93.7\%) and MIS TLIF (92.7\%) was not significant $(p=0.43)$. More importantly, the authors reported a statistically significant reduction in hospital stay $(p<0.001)$ and time to ambulation $(p<0.001)$ compared with MIS TLIF.

To summarize, although existing studies differ in terms of study design and measured outcome variables, most studies agree that biportal ELIF has favorable clinical outcomes and fusion rates. Moreover biportal ELIF leads to lesser intra-operative blood loss and reduced immediate postoperative back pain compared to open PLIF/MIS TLIF, the findings of which are summarized in Table 2.

\section{Advantages of Biportal ELIF}

\section{1) Minimal Invasiveness}

Biportal ELIF can minimize paraspinal muscle damage, as multifidus muscle attachments can be preserved via the transmuscular approach. Further, muscle ischemia can be reduced, as a tubular retractor is not used between the paraspinal muscles in biportal ELIF $^{5,11,16)}$. By employing a semitubular retractor and accessing through the transmuscular surgical approach, biportal ELIF causes less paraspinal muscle injury (Figue 1C). Especially, biportal ELIF can preserve the paraspinal muscles of the contralateral side using a unilateral approach.

Due to the minimal invasiveness of biportal ELIF, intraoperative blood loss and postoperative back pain can be reduced in comparison to patients who undergo open PLIF/MIS TLIF ${ }^{5,11,16)}$. These advantages of biportal ELIF can possibly result in quicker recovery and an earlier return to daily life following surgery. Three comparative studies on open PLIF/MIS TLIF demonstrated that immediate postoperative back pain was significantly lower than it was for open PLIF/MIS TLIF. Further, these studies demonstrated favorable outcomes comparable to open PLIF/MIS TLIF along with the additional benefits associated with reduced blood loss, early ambulation, and reductions in hospital stay ${ }^{5,11,16)}$. However, in order to accurately compare muscle injury with other fusion techniques, further research on radiological and laboratory is required.

\section{2) Direct Decompression and Cage Insertion with Magnified Vision and Less Limitation}

With biportal ELIF, independent movement of the endoscope 
and surgical instruments is possible, and the working portal is used only for surgical instruments, though not a tubular retractor ${ }^{6}$. As a consequence, complete direct decompression of the central canal, lateral recess, and foraminal area with less motion and vision limitation is possible (Figure 2$)^{4-6,10,11,16)}$. Specifically, decompression of the contralateral exiting nerve root is relatively easy through the contralateral sublaminar approach (Figure 2D). Furthermore, biportal ELIF provides good operative vision with a high magnification endoscopic view and a clearing view by continuous irrigation for safe and complete decompression ${ }^{4,10,16)}$.

Large-sized cages for biportal ELIF can be inserted into the disc space, because the cage passes through the working portal and not through the working cannula ${ }^{4-6,10,11,16)}$. As a result, indirect decompression can be achieved with large-sized cages through a reduction of spondylolisthesis and restoration of the disc space. Performing contralateral facetectomy through the contralateral sublaminar approach is crucial for the reduction of listhesis and the restoration of disc height (Figure $3 \mathrm{~B}$ ) ${ }^{4}$. Since during cage insertion in biportal ELIF a blind space is made, a retractor designed to securely protect the thecal sac and traversing root can prevent nerve injury under fluoroscopic guidance (Figure $5 \mathrm{~B})^{16)}$.

\section{3) Endplate preparation and Fusion Rate}

Endplate preparation is essential for solid fusion in lumbar interbody fusion. Incomplete endplate preparation or endplate injury can lead to cage subsidence or fusion failure. However, complete endplate preparation can be difficult because it is not identified directly in conventional lumbar interbody fusion ${ }^{10)}$. One of the biggest advantages of biportal ELIF involves endplate preparation. Biportal ELIF can allow meticulous endplate preparation, as endplate preparation can be performed under endoscopic guidance (Figure 4$)^{5,6,10,16)}$. The cartilaginous endplate can be dislodged from the osseous endplate using freer elevator. Also, endplate preparation under endoscopic guidance can reduce the chance of endplate injury, which can prevent cage subsidence. Moreover, surgeons can determine when endplate preparation is complete under a magnified endoscopic view.

In total, 3 articles examined the fusion rate after biporal ELIF with only simple radiography. These studies that compared biportal ELIF with open PLIF/MIS TLIF presented similar fusion rates at 12 months of follow-up, while the fusion rates for both groups were not significantly different. However, Park et al. ${ }^{16)}$ reported a statistical trend that fewer patients had definite fusion with biportal ELIF (71\%) than with open PLIF (83\%), along with significantly fewer cases of definite fusion with biportal ELIF than with open PLIF. That result suggests that biportal ELIF has further to go in terms of improving the fusion rate. No study on biportal ELIF reported the long-term results for fusion rate, and solid fusion was only evaluated with simple radiography. As a result, it is still too early to fully validate the fusion rate for biportal ELIF. Future evaluation by computed tomography (CT) in longterm follow-up is necessary to estimate solid fusion.

Given biportal ELIF has a small surgical area, fusion with ELIF has the potential for adequate bone grafting and graft site preparation to allow arthrodesis to occur. As a result, several efforts are underway to improve the fusion rate for biportal ELIF. First, meticulous endplate preparation is crucial to successful arthrodesis. As described above, meticulous endplate preparation is possible in biportal ELIF as compared to other LIF techniques. Commonly, 70-80\% of the disc space can be prepared for fusion with biportal ELIF. Second, prior to cage insertion, many allogenous or autogenous bone chips can be placed into the disc space with a specialized funnel. It is necessary to stop saline irrigation during the insertion of fusion materials in order to prevent bone chip loss. The fusion rate is increased by using a demineralized bone matrix. Third, generally, a single TUF cage is employed. However, two PLIF cages can be inserted ${ }^{4}\{\mathrm{Heo}, 2020$ \#39\}. After cage insertion, it is placed in the intervertebral space with a cage impactor to ensure it is located in the centre-anterior portion of the disc space. To prevent cage subsidence, it should rest on the stronger anterior ring apophysis rather than just on the soft central cancellous portion. Finally, osteoporosis can result in the pedicle screws becoming loose, which subsequently would reduce axial compressive strength ${ }^{15}$. Therefore, it is necessary to determine the BMD of each patient prior to surgery and to provide antiosteoporosis drugs to the patients.

\section{4) Learning Curve}

Previous endoscopic techniques have been difficult to improve due to restricted vision, motion, and unfamiliar surgical views ${ }^{3,7)}$. On the other hand, in biportal ELIF, as the working portal is used only to manipulate spinal instruments, the surgery can be performed using instruments without significant limitations. And it has an advantage with a magnified and clear surgical view under fluid-medium endoscopic guidance. Futher, biportal ELIF can provide a similar surgical view with a microsurgical view, which is familiar to spine surgeons, which may help in learning this technique ${ }^{6,16)}$.

\section{Limitations of Biportal ELIF}

\section{1) Limited Indications}

Biportal ELIF can be adapted to many situations due to its wide range of indications similar to conventional fusion techniques. However, biportal ELIF remains a technically challenging and complex procedure. Complicated cases, such as deformity correction, reduction of high-grade spondylolisthesis, spondyloptosis, and spondylolisthesis with a fused segment are not available in biportal ELIF,16). As the technique develops, indications for biportal ELIF are expected to widen in the future.

\section{2) Complications}

Reported complications for biportal ELIF have included postoperative hematoma, dural tear, infection, and nerve root injury $5,6,10,11,16)$. Three studies on biportal ELIF have compared it to complications arising from open PLIF/MIS TLIF, and no significant differences in complication rate were found ${ }^{5,11,16)}$. A dural tear is a central concern for biportal ELIF. In the 5 articles on biportal ELIF, there were 7 cases of dural tears out of 209 
patients (3.3\%). The sizes of most dural tears were not large enough to directly suture, but the dural tear could be treated with a fibrin collagen patch (TachoComb) and bed rest for 5 to 7 days. However, if the size of dural tear is larger than 10 $\mathrm{mm}$, a dural suture under endoscopy or conversion to microscopic surgery is recommended ${ }^{6,16)}$.

Although no water-related complications have been reported in previous articles about bipotal ELIF, caution is necessary for fluid output as biportal ELIF is a fluid-medium surgery. Otherwise, water-related complications, such as headache, seizure, and retroperitoneal fluid collection, are possible. To maintain fluid output, a retractor, like a semitubular retractor, should be used to prevent water-related complications.

\section{3) Operation Time}

Standardly, the mean operation time for biportal ELIF is longer than it is for open PLIF/MIS TLIF, ${ }^{5,16)}$. Biportal ELIF using one hand while the other hand holds an endoscope can increase the operation time. Also, meticulous endplate preparation can increase the operation time ${ }^{16)}$. Therefore, performing multi-level biportal ELIF can likely increase operative times and radiation exposure compared to other fusion modalities. Biportal ELIF needs further technological development to shorten operation times.

\section{The Current Level of Evidence}

Current evidence on the effectiveness of biportal ELIF remains limited. Only a few case series or case-control studies with short follow-up periods have been published on biportal ELIF. Despite the popularity of biportal ELIF, no randomized controlled studies have been done to compare it with conventional surgeries. Further prospective studies with a long follow-up period are needed to prove the reliability of biportal ELIF.

\section{CONCLUSION}

This review article indicates that biportal ELIF is as effective as open PLIF/MIS TLIF in improving fusion rate and clinical outcomes. Biportal ELIF has distinct advantages over open PLIF/MIS TLIF: (1) reduced intraoperative blood loss, (2) less immediate postoperative back pain, and (3) meticulous endplate preparation under endoscopic guidance. Even though biportal ELIF has demonstrated several advantages, limitations are also associated with the technique, including longer operation times, limited indication, and a lack of multi-center randomized controlled trials that directly compare other fusion techniques with biportal ELIF. Well-designed studies with a long-term follow-up and prospective randomized controlled trials should be conducted to further establish the effectiveness and safety of biportal ELIF.

\section{CONFLICT OF INTEREST}

No potential conflict of interest relevant to this article.

\section{REFERENCES}

1. Ao S, Zheng W, Wu J, Tang Y, Zhang C, Zhou Y, et al.: Comparison of Preliminary clinical outcomes between percutaneous endoscopic and minimally invasive transforaminal lumbar interbody fusion for lumbar degenerative diseases in a tertiary hospital: Is percutaneous endoscopic procedure superior to MIS-TLIF? A prospective cohort study. Int J Surg 76:136-143, 2020

2. Gejo R, Matsui H, Kawaguchi Y, Ishihara H, Tsuji H: Serial changes in trunk muscle performance after posterior lumbar surgery. Spine (Phila Pa 1976) 24:1023-1028, 1999

3. Gibson JNA, Subramanian AS, Scott CEH: A randomised controlled trial of transforaminal endoscopic discectomy vs microdiscectomy. Eur Spine J 26:847-856, 2017

4. Heo DH, Hong YH, Lee DC, Chung HJ, Park CK: Technique of Biportal Endoscopic Transforaminal Lumbar Interbody Fusion. Neurospine 17:S129-S137, 2020

5. Heo DH, Park CK: Clinical results of percutaneous biportal endoscopic lumbar interbody fusion with application of enhanced recovery after surgery. Neurosurg Focus 46:E18, 2019

6. Heo DH, Son SK, Eum JH, Park CK: Fully endoscopic lumbar interbody fusion using a percutaneous unilateral biportal endoscopic technique: technical note and preliminary clinical results. Neurosurg Focus 43:E8, 2017

7. Hsu HT, Chang SJ, Yang SS, Chai CL: Learning curve of fullendoscopic lumbar discectomy. Eur Spine J 22:727-733, 2013

8. Jin M, Zhang J, Shao H, Liu J, Huang Y: Percutaneous Transforaminal Endoscopic Lumbar Interbody Fusion for Degenerative Lumbar Diseases: A Consecutive Case Series with Mean 2-Year Follow-Up. Pain Physician 23:165-174, 2020

9. Kawaguchi Y, Yabuki S, Styf J, Olmarker K, Rydevik B, Matsui $\mathrm{H}$, et al.: Back muscle injury after posterior lumbar spine surgery. Topographic evaluation of intramuscular pressure and blood flow in the porcine back muscle during surgery. Spine (Phila Pa 1976) 21:2683-2688, 1996

10. Kim JE, Choi DJ: Biportal Endoscopic Transforaminal Lumbar Interbody Fusion with Arthroscopy. Clin Orthop Surg 10:248252, 2018

11. Kim JE, Yoo HS, Choi DJ, Park EJ, Jee SM: Comparison of Minimal Invasive Versus Biportal Endoscopic Transforaminal Lumbar Interbody Fusion for Single-level Lumbar Disease. Clin Spine Surg, 2020

12. Kolcun JPG, Brusko GD, Basil GW, Epstein R, Wang MY: Endoscopic transforaminal lumbar interbody fusion without general anesthesia: operative and clinical outcomes in 100 consecutive patients with a minimum 1-year follow-up. Neurosurg Focus 46: E14, 2019

13. Lee KH, Yue WM, Yeo W, Soeharno H, Tan SB: Cinical and radiological outcomes of open versus minimally invasive transforaminal lumbar interbody fusion. Eur Spine J 21:2265-2270, 2012

14. Ng LC, Tafazal S, Sell P: The effect of duration of symptoms on standard outcome measures in the surgical treatment of spinal stenosis. Eur Spine J 16:199-206, 2007

15. Park MK, Kim KT, Bang WS, Cho DC, Sung JK, Lee YS, et al.: Risk factors for cage migration and cage retropulsion following transforaminal lumbar interbody fusion. Spine J 19:437-447, 2019 
16. Park MK, Park SA, Son SK, Park WW, Choi SH: Clinical and radiological outcomes of unilateral biportal endoscopic lumbar interbody fusion (ULIF) compared with conventional posterior lumbar interbody fusion (PLIF): 1-year follow-up. Neurosurg Rev 42: 753-761, 2019

17. Shen J: Fully Endoscopic Lumbar Laminectomy and Transforaminal Lumbar Interbody Fusion Under Local Anesthesia with Conscious Sedation: A Case Series. World Neurosurg 127:e745e750, 2019

18. Sihvonen T, Herno A, Paljarvi L, Airaksinen O, Partanen J, Tapaninaho A: Local denervation atrophy of paraspinal muscles in postoperative failed back syndrome. Spine (Phila Pa 1976) 18: 575-581, 1993

19. Styf JR, Willen J: The effects of external compression by three different retractors on pressure in the erector spine muscles during and after posterior lumbar spine surgery in humans. Spine (Phila Pa 1976) 23:354-358, 1998

20. Wang J, Zhou Y, Zhang ZF, Li CQ, Zheng WJ, Liu J: Compari- son of one-level minimally invasive and open transforaminal lumbar interbody fusion in degenerative and isthmic spondylolisthesis grades 1 and 2. Eur Spine J 19:1780-1784, 2010

21. Wang MY, Grossman J: Endoscopic minimally invasive transforaminal interbody fusion without general anesthesia: Initial clinical experience with 1-year follow-up. Neurosurg Focus 40:E13, 2016

22. Wu J, Liu H, Ao S, Zheng W, Li C, Li H, et al.: Percutaneous Endoscopic Lumbar Interbody Fusion: Technical Note and Preliminary Clinical Experience with 2-Year Follow-Up. Biomed Res Int 2018:5806037, 2018

23. Wu PH, Kim HS, Lee YJ, Kim DH, Lee JH, Jeon JB, et al.: Uniportal Full Endoscopic Posterolateral Transforaminal Lumbar Interbody Fusion with Endoscopic Disc Drilling Preparation Technique for Symptomatic Foraminal Stenosis Secondary to Severe Collapsed Disc Space: A Clinical and Computer Tomographic Study with Technical Note. Brain Sci 10, 2020 